\title{
Pain mapping: A mechanisms-oriented protocol for the assessment of chronic pelvic pain and urogenital pain syndromes
}

\author{
(D) MAREK JANTOS \\ Adelaide, Australia
}

\section{ABSTRACT}

Objective: Chronic pelvic pain (CPP) and various pelvic dysfunctions are best assessed by consultants with knowledge and training in myofascial pain. The prevalence of myofascial pain is recognised but what is needed is a validated protocol to guide physical examination of pelvic structures. Pain mapping was developed to assist with localising active and passive sources of pain, evaluating its severity, temporal characteristics, topography and mechanisms and guiding therapy.

Materials and Methods: This is a prospective study involving the pain mapping of 320 female volunteers, consisting of women diagnosed with chronic urogenital pain (CUP), and of a comparison and control group. The protocol uses three pain maps to guide assessment of the external urogenital area, internal pelvic floor structures and paraurethral region, and follows an established strategy to maintain consistency.

Results: The mean age of the CUP group was 34.7 \pm 12.1 years; $31.6 \pm 10.1$ for the gynaecology comparison group; and $35.5 \pm 11.5$ for the control group. There were no significant differences in age or parity, the groups were well matched for statistical comparison. The highest pain scores from Map A were noted around the vestibule and urethral meatus; from Map B included all of the internal pelvic structures tested; and from Map C all points were painful and accounted for the highest scores of all the points mapped. Logistic regression analysis identified two points from each of the three maps (a total of six points), that provide $94 \%$ accuracy in the diagnosis of chronic urogenital pain syndrome.

Conclusion: The pain mapping study demonstrates the benefits of using an established protocol for localising and assessing pelvic pain. The results highlight the role of peripheral mechanisms, in the form of myofascial changes associated with pain and organ dysfunction. The paraurethral area appears to be the primary generator of CUP symptoms and diagnostically is the most reliable in differentiating between CUP cases and asymptomatic controls. As an anatomical region the paraurethral area is an overlooked source of pain and rarely tested during diagnostic assessments.

Keywords: Bladder pain syndrome; chronic pelvic pain; chronic urogenital pain; myofascial pain; pain mapping; vulvodynia

\section{INTRODUCTION}

The diagnosis and management of chronic pelvic pain (CPP) requires extensive knowledge and expertise. The pelvis is a welldefined region but anatomically complex. It consists of bones, multiple layers of muscle and fascia and housing within its bony structure various organs that belong to biological systems that converge in its confines. As a result CPP is difficult to localise. Any persistent pain that is experienced between the umbilicus 
and the upper thighs can be classified as CPP, even if its origin is unknown and cause difficult to identify. The aim of this study is to further establish the validity of a pain mapping protocol for the assessment of CPP.

CPP is defined as a recurrent or persistent pain, unrelated to menstruation, intercourse or pregnancy, that lasts at least six months and causes functional impairment requiring medical or surgical treatment., ${ }^{1,2}$ When potential organic pathologies are excluded and pain persists and has a life-altering impact, it is classified as a chronic pain syndrome. Without knowing the pathogenesis and mechanisms of such pain syndromes their management is difficult. With the prevalence of CPP syndromes in the general population estimated to be as high as 39\% it poses a challenge to clinical practice. ${ }^{3}$ These pain disorders account for almost half of all laparoscopies and a significant number of hysterectomies.

Myofascial pain is widely recognised as a source of CPP, but this has not always been the case. Three decades ago, the prevalence of myofascial disorders in CPP was estimated to be around eight percent, while current estimates place it at $85-90 \% .4,5$ The ability to identify myofascial pain is contingent on the training of the health consultant. If the patient is seen in a primary care setting only $30 \%$ of CPP cases receive a diagnosis of myofascial pain syndrome, but if seen in a dedicated pain centre the figure is $85 \%$ or higher. ${ }^{5,6}$ Very few physicians have been trained to assess pain of muscle and fascial origin. ${ }^{7}$ Without this training, gynaecologists, urologists and proctologists are left with the only option of managing pain according to the standard protocols available to them. ${ }^{7,8}$ This highlights the dual need of training and of protocols for in-clinic assessments.

Myofascial pain necessitates a physical examination of all pelvic structures; muscles, joints, ligaments, fascia and viscera; and must include a functional appraisal of the biomechanics of pelvic soft tissue. ${ }^{9}$ To date clinical assessments have relied on the cotton swab test, commonly referred to as the Kaufman Q-tip test. ${ }^{10}$ This is especially the case with chronic urogenital pain (CUP) conditions, which affect the reproductive and urinary systems. The Q-tip test has been used for clinical and research purposes for over 30 years. Its focus is limited to examining tenderness within the vulvar vestibule, as originally proposed by Friedrich. ${ }^{11}$ This falls short of the recommendations of consensus statements advocating bilateral palpation of muscles, and a functional assessment of pelvic structures. ${ }^{12}$

In the past CPP was commonly attributed to end-organs such as bladder, bowel and external genitalia, but more recently the focus has shifted to myofascial changes and high-tone pelvic muscle dysfunction. ${ }^{13-16}$ Another cause, that was often suspected, was endometriosis. However, recent studies found no correlation between level of disease and severity of pain, suggesting that

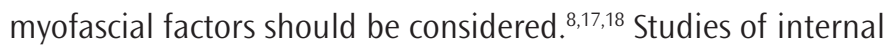
pelvic muscles show that tenderness best differentiates between symptomatic and asymptomatic women. ${ }^{19,20}$ Nulliparous women with no lower urogenital tract symptoms report no tenderness, while $94 \%$ of women with CUP report clinically significant levels of tenderness.

To enable consistency and precision of myofascial assessment there is a need for a standardised protocol. An extensive literature review of assessment procedures notes that "a standardised and reproducible protocol... does not currently exist, and few providers evaluate for pelvic floor myofascial pain even in patients presenting with pelvic pain..."?

A pain mapping protocol, developed by the author and his associates, has been described in literature and its validity is further tested in this study. ${ }^{21,22}$ It uses predefined examination points and a clearly outlined strategy to ensure consistency and reliability of the assessment procedure. Both active and passive sources of pain can be identified and evaluated in terms of severity, temporal characteristics and topography. In reproducing pain and symptoms, the process validates the patients reports and provides an evidence base for the planning of treatment. Importantly, pain mapping is focused on the complex question of "where the pain is coming from," not on the basal one of "where is the pain?" It shifts the focus from an oversimplified topographical approach using body forms, to one localising the source of pain and examining the peripheral mechanisms involved.

Three pain maps are used in this study, each one was developed on the basis of clinical work, literature reports and cadaver dissections. ${ }^{22}$ The first pain map focuses on the external urogenital area (Map A), in particular on the superficial tissue of the vulva, thought to be related to chronic vulvar pain. The second pain map looks at the deep fascia and pelvic muscles (Map B), and the third map (Map C), developed by the author and his associates, examines the paraurethral and bladder area, thought to be the source of bladder pain.

\section{MATERIALS AND METHODS}

This is a prospective study based on the pain mapping of 320 consecutive volunteers attending a multidisciplinary women's health clinic in Lublin, Poland. The aim of the study was to test a standardised pain mapping assessment protocol to localise the source of pain; assess its severity, quality and topography in women diagnosed with CUP syndromes; and to compare their 
pain profiles with the profiles of women presenting with other gynaecological problems but no pain, and with asymptomatic women who were part of a control group.

Participants consisted of volunteers who agreed to participate after receiving an overview of the study, its aims and methods. The inclusion criteria stipulated that the participants must be 18 years of age or older and able to provide consent. The exclusion criteria included pregnancy, birth or pelvic surgical procedure in the last three months, a history of major reconstructive surgery, a known diagnosis of active endometrial disease or any other concurrent illness.

Based on the medical diagnoses volunteers were allocated to the subgroup shown in Figure 1.

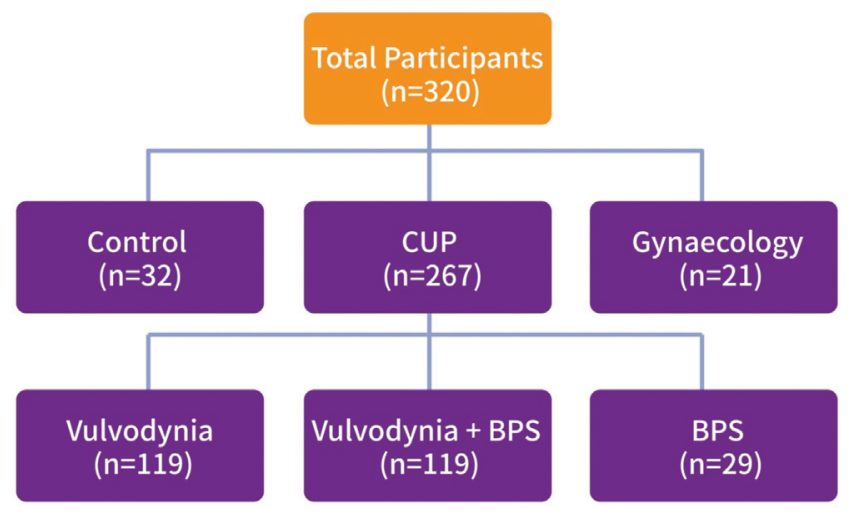

Figure 1. Study subgroup comparison structure. CUP: Chronic urogenital pain, BPS: Bladder pain syndrome

The Control group consisted of women attending the clinic for routine PAP smear surveillance only and had no history of gynecological, urological or CUP symptoms. The women in the Gynecology group presented with gynecological problems that included PAP smear abnormalities, pelvic inflammatory disease, lichens sclerosis or polycystic ovarian syndrome, but no history of pain. The CUP group was made up of those diagnosed with vulvodynia or bladder pain syndrome (BPS) or having a dual diagnosis of both vulvodynia and BPS. Instruments used in the study included a consent form and a study questionnaire and three pain maps for recording information derived from the physical examination. Map A was used for examining the external urogenital area (27 Points); Map B for the internal examination of pelvic muscles (15 points); and Map C for palpation of the paraurethral area (12 points). These three maps are shown in Figure 2A-C.

The assessment of each point required three items of information: a pain severity score using a verbally

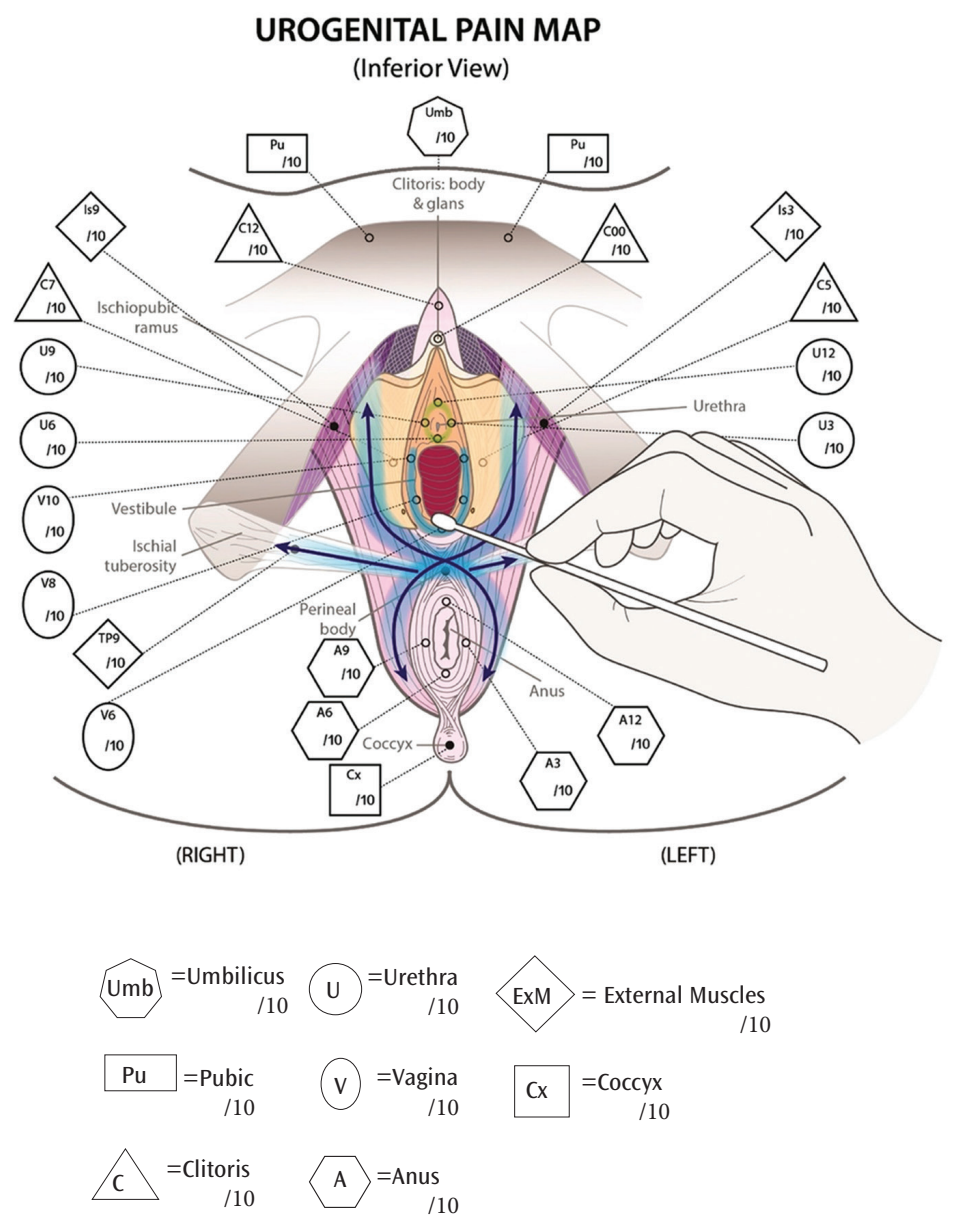

Figure 2 A. Map A-External Urogenital Pain Map, identifying external assessment points

administered 0-10 numerical rating scale (NRS), where zero is no pain and ten the most severe pain experienced. ${ }^{23} \mathrm{~A}$ list of pain descriptors using adjectives from a modified McGill Pain Questionnaire list $^{24}$ and a record of the patients account of the spatial distribution of pain as experienced at the time of examination.

The examiner and her assistants were well versed in the identification of the mapping points, having participated in several earlier pain mapping studies. A more detailed description of the pain mapping protocol, its development and validation are discussed elsewhere in literature. ${ }^{25}$ The examination procedure consisted of a medical exam performed by a tertiary specialist (a female gynaecologist) to exclude any anatomical problems or current infections and diseases, which was followed by the pain mapping assessment. Prior to pain mapping, participants were asked to empty their bladder. The pain mapping was carried out in a lithotomy position in a gynaecology chair. All points were examined 


\section{PELVIC MUSCLE PAIN MAP}

(Superior View)

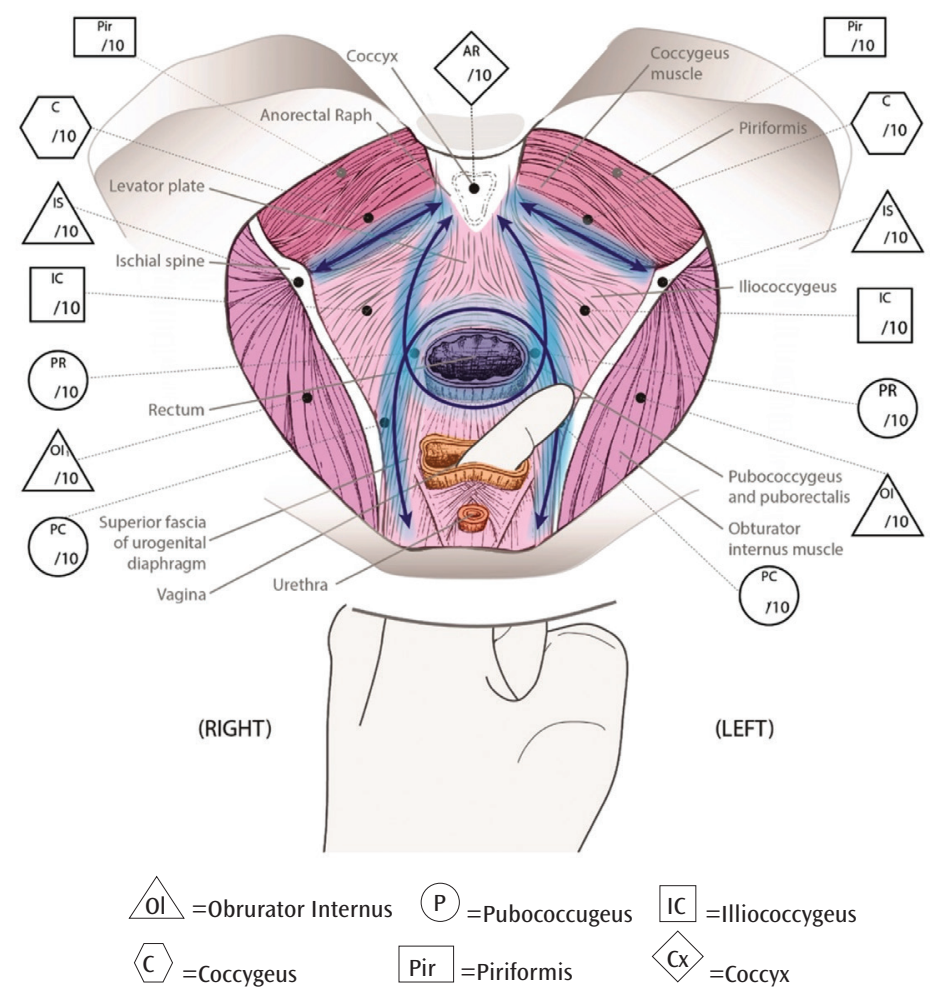

Figure 2 B. Map B-Internal pelvic structures assessed

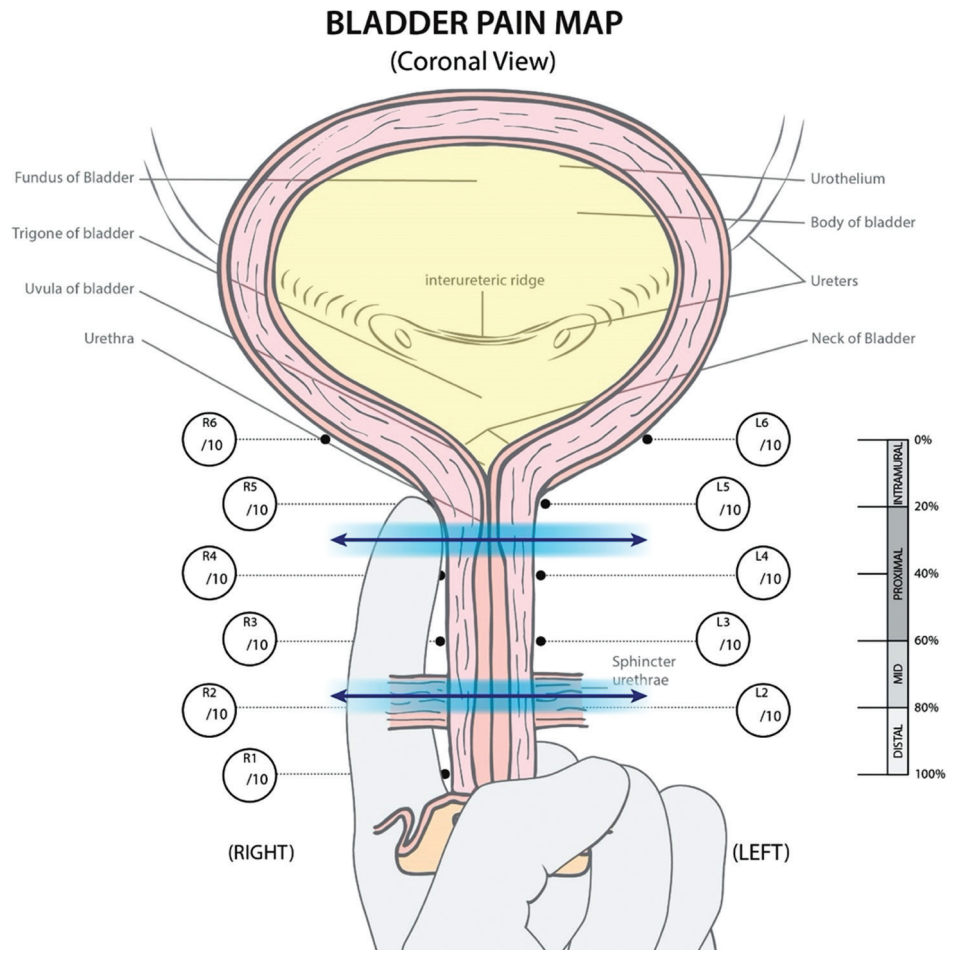

Figure 2 C. Map C-identifying paraurethral assessment points by gloved hand using either digital pressure or Q-tip for the vestibular and anal points. The Q-tip was a fine $15 \mathrm{~cm}$ long examination stick, lightly moistened with hypoallergenic, pure paraffin ointment. The pressure for digital palpation was 0.4$0.5 \mathrm{~kg} / \mathrm{cm}^{2}$ and for Q-tip testing $0.1-0.2 \mathrm{~kg} / \mathrm{cm}^{2}$ as per earlier studies. ${ }^{22}$

Points examined on Map A included the pubis, transverse and perineal points (using digital pressure), vestibule, urethra, clitoris and anus (using Q-tip), all points were marked on the basis of a perineal/pelvic clock as shown in Figure 2A. The pelvic floor assessment in Map B included bilateral examination of all of the pelvic muscles, and ischial spine. This was carried out by the examiner using a single digit inserted into the vaginal canal as shown in Figure 2B. Each participant was instructed to relax the pelvic muscle by selfdilating with the assistance of a diaphragmatic breathing technique, to ensure a more comfortable digital insertion and assessment of pelvic points. In instances of extreme sensitivity, the participants were given the option of having $10 \%$ lidocaine gel applied to the introital area. The gel was used only upon completion of Map A and prior to Map B and $C$ assessments. The first task prior to palpation of pelvic points was a simple test to evaluate pelvic muscle strength, using the Oxford scale.

CUP is associated with non-relaxing pelvic muscles, functional shortening of muscles and a general contracture, associated with loss of muscle elasticity leading to narrowing the central hiatus. These observations were always noted in the course of pelvic assessment. Next all of the points were palpated, using the palmar side of the finger, and data recorded. Map C was completed by rotating the examiners hand so that the palmar side faced the urethra and bladder. Each of the six points on the left and then six points on the right, just lateral to the urethra, were palpated with the pointer finger, starting with the $100^{\text {th }}$ percentile, then moving to the $80^{\text {th }}, 60^{\text {th }}$, $40^{\text {th }}, 20^{\text {th }}$ and 0 percentile, level with the vesical neck, as shown in Figure $2 \mathrm{C}$.

The data from pain mapping provided an individualized pain profile for each participant. The results were reviewed with each individual. Additional assessments were carried out by members of the multidisciplinary team where each participant underwent a general physiotherapy and postural assessment, and a psychology assessment. For the purposes of this report, only pain mapping data will be analysed.

Ethics approval was granted by the Bioethics Commission of the Medical University of Lublin for routine clinical assessment and collection of pain mapping information (approval no: KE0254/226/2014, date: 26.06.2014). Each participant's anonymity 
was maintained by complete de-identification of data prior to data analysis.

\section{Statistical Analysis}

A range of statistical analyses were used, including, t-tests for comparison of two means, chi-square tests for independence between categorical variables, Pearson correlation coefficient, analyses of variance and logistic regression analyses.

\section{RESULTS}

The mean age of the CUP group was $34.7 \pm 12.1$ years; $31.6 \pm 10.1$ for the Gynaecology group; and $35.5 \pm 11.5$ for the Control. There was no significant difference in the mean ages of the CUP, Gynaecology group and Control group. The age distribution for the CUP group showed that prevalence in this study peaked around age 25 . Parity for each group was similar $(0.7 \pm 1.0)$. With no significant differences in age or parity, the groups were well matched for statistical comparison.

The first stage of data analysis focused on comparing mean pain scores for each point for the CUP groups, with the mean pain scores of the Gynaecology and Control groups. The summary of the data is presented in Figure 3. For purposes of this study pain mapping scores on any of the points that were greater or equal to $2 / 10(\geq=2)$ were considered clinically significant.

The severity of pain ratings for all points in the CUP group is shown in Figure 4.
Thirteen points on Map A and all points on Map B and Map $C$ differentiated between pain groups and controls. With the remaining points on Map A there was no significant pain reported. These points are of no clinical or diagnostic value.

CUP group ratings were analysed by anatomical region. On Map A, the CUP group as a whole, identified the vestibular points, in particular the posterior fourchette, as being most painful, followed by the urethral points, with no significant pain in the clitoral, umbilical and anal region. On Map B, with the exception of the deep piriformis muscle, all points were rated as significantly painful. On Map C three trends were noted; the paraurethral points were assigned the highest pain ratings of all three maps by all of the CUP women; pain scores progressively increased from the distal to the proximal portion of the urethra; and pain on the left side of the urethra was consistently higher than on the right side of the urethra.

A between groups comparison was made on the three maps. In the control group the mean pain scores across all points were less than two, therefore clinically not significant. For women in the Control group, the highest mean pain score reported was on Map A point V4 (left vestibular region-1.3/10). The control group also reported some pain on other vestibular points (AV 2,4,6,8,10), but the pain scores were less than 2 and were not clinically significant. From the data it is clear that pain was not a feature of the asymptomatic group on any of

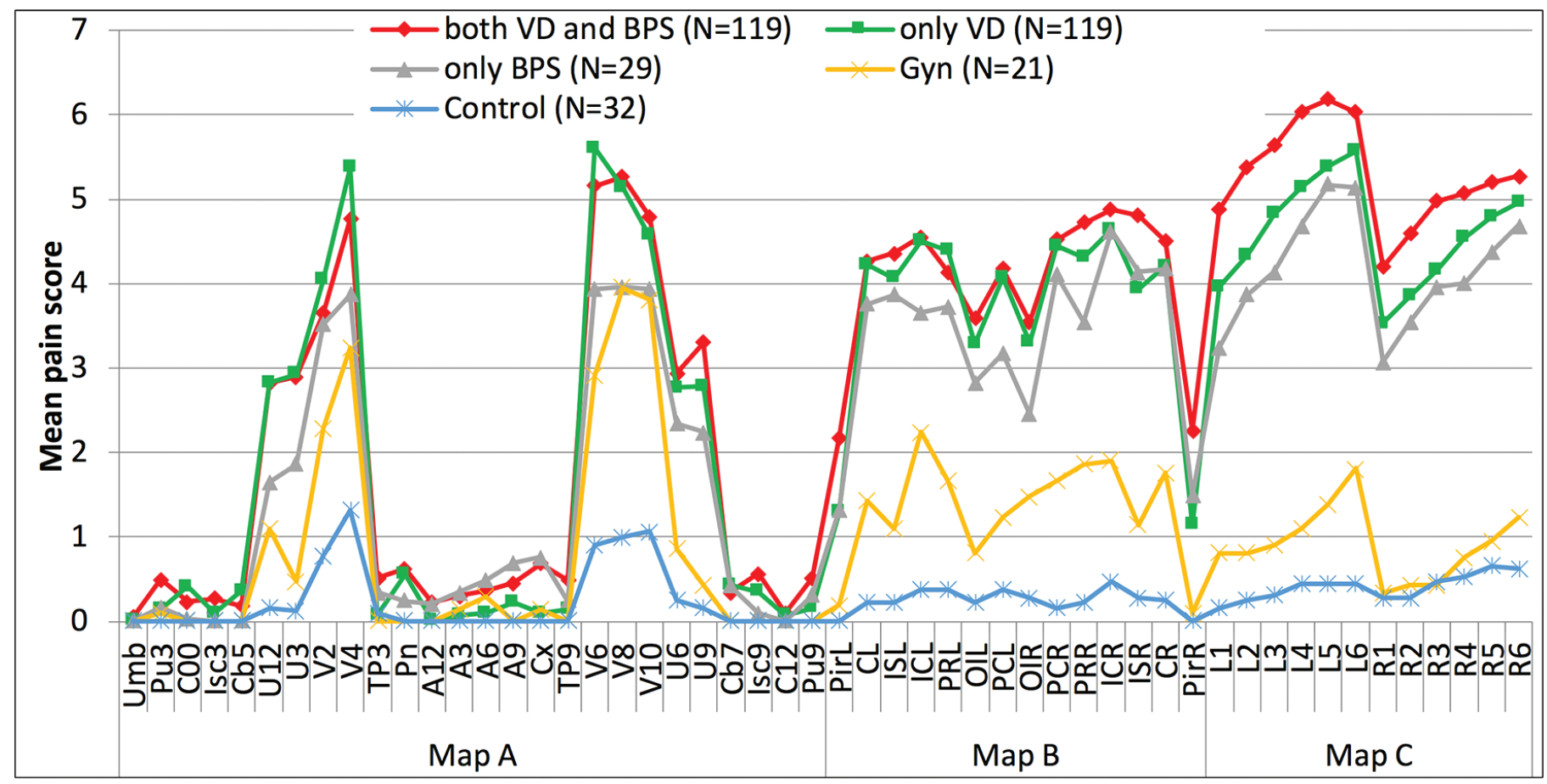

Figure 3. Mean pain scores for all points on Map A, B and C, across all groups. VD: Vulvodynia; BPS: Bladder pain syndrome 


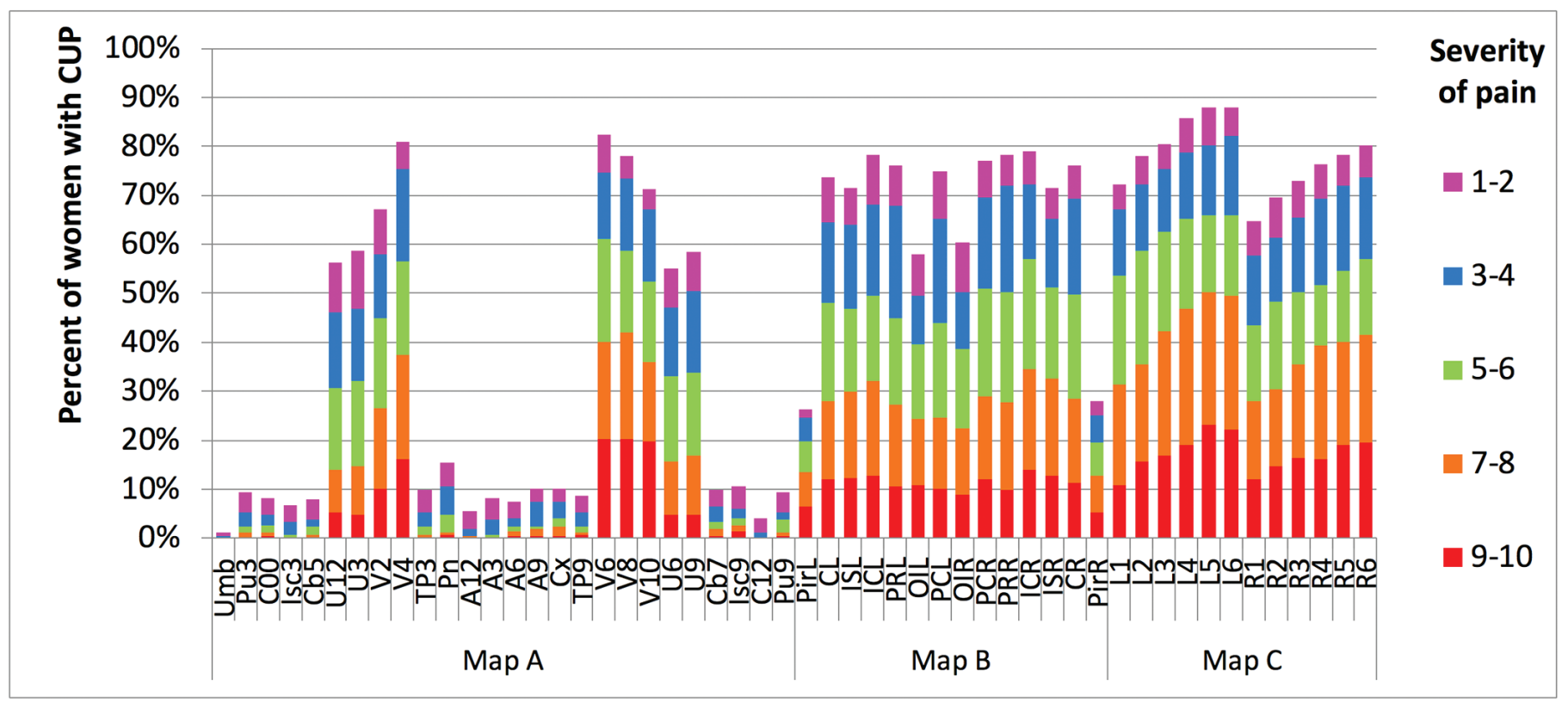

Figure 4. Severity of pain ratings for each point on the three maps

the points examined. On Map A, significant differences were noted between the CUP, gynaecology and control groups on all of the urethral and vestibular points. On Map B and C all of the points palpated showed significant differences between CUP, gynaecology and control group.

Pain scores of the CUP subgroups of vulvodynia and BPS were compared. On Map A, women with vulvodynia only, reported higher scores than those with vulvodynia and BPS or BPS only. On Map B and C women with the dual diagnosis of vulvodynia and BPS reported highest scores with no significant difference between the two subgroups. Pain mapping reliably differentiated between vulvodynia and asymptomatic controls. However, the sub-classification of vulvodynia into provoked and spontaneous showed no significant differences between these two groups.

Logistic regression analysis of pain scores from the three pain maps using four different models identified the most reliable diagnostic points. In model 1, comparing the pain data from CUP and control subjects, the points most closely and reliably associated with a diagnosis of CUP are AV6, AV10 and AU9. In Model 2, 3 and 4, if the pain scores on each pain map are considered as independent variables, then on Map A, points AV6 and AU 9 are the most reliable predictors, and on Map B points BISL and BPRR, and on Map C the points CL2 and CL5 are the most reliable for the diagnosis of CUP.

For the CUP women, the mean Oxford scale score was 2.6 ( \pm 1.9$)$ and was significantly lower, and by implication the muscles appeared weaker, when compared with the mean score of the
Control group, which was $3.5( \pm 1.7)$. The CUP group though reporting the highest pain scores had weaker pelvic muscles.

The referred pain patterns (pain topography) were recorded and analysed. In the CUP group, the points on Map A that were reported as painful, produced localised pain only, this was evident in the posterior fourchette and around the urethral meatus and is shown in Figure 5A. None of these points referred pain to distant locations. Points on Map B, in

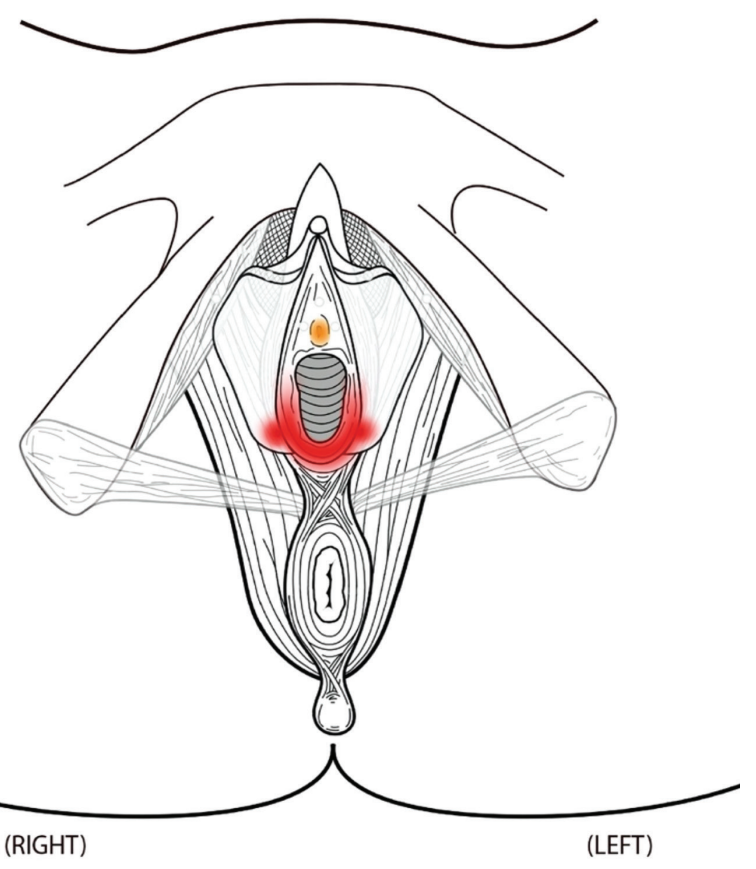

Figure $5 \mathrm{~A}$. Referred pain from Map A 
addition to localised tenderness, referred pain to more distant points, including abdomen, hips, lower back and groin, with some points in the posterior section of the pelvis reproducing faecal urge and irritation of the bladder. The referred pain areas are shown in Figure 5B. The referred pain patterns from Map C are most complex and extensive as shown in Figure 5C. Pain in the paraurethral area was experienced locally upon palpation, in addition pain was referred to the umbilical region, the right and left iliac region, epigastric regions

\section{DISTRIBUTION OF PAIN FROM PELVIC MUSCLE AREA}

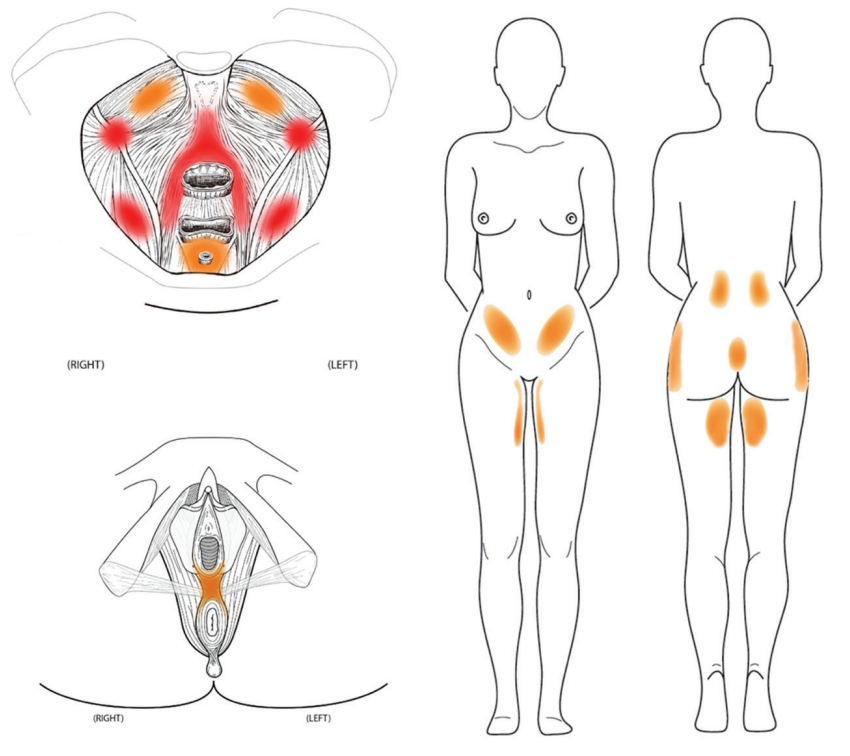

Figure 5 B. Referred pain from Map B

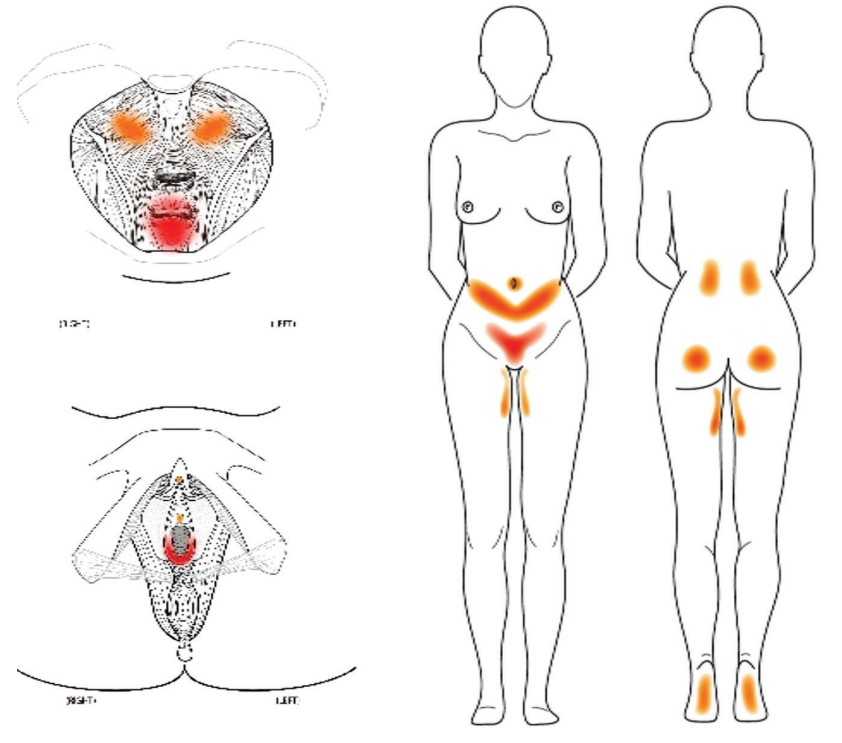

Figure 5 C. Referred pain from Map C (reproducing sensation of suprapubic pressure, lower back, groin and soles of the feet (reported as a burning sensation).

In addition, the paraurethral points reproduced bladder urge of varying severity, clitoral pain (even in women who did not report symptoms of clitoral pain) and sensations of arousal in women who presented with persistent arousal disorder symptoms. Paraurethral pain in the majority of women existed as passive pain and was reproduced during physical examination. None of the CUP women during initial screening complained of pain in the paraurethral area.

\section{DISCUSSION}

This is the largest pain mapping study undertaken to date. It is based on data derived from a sample of 320 symptomatic and asymptomatic volunteers. The large sample size is one of the strengths of this study. The pain mapping protocol focussed on the physical examination of a series of predefined points as shown in the three pain maps in Figure 2A-C (Map A, B and $C$ ). The research is original and first to map pain arising from the paraurethral area. It provides unprecedented insight linking the origins of pain with symptoms reported by women diagnosed with CUP.

The term "pain mapping," refers to the process of localizing pain, and establishing a relationship between the source of pain and the symptoms experienced by each individual. 21,22 Pain mapping data also provides a pain profile of the CUP group and reliably differentiates between symptomatic and asymptomatic women. From the analysis it is evident that pain is not a characteristic of asymptomatic women but is a defining feature of CPP and CUP. ${ }^{20}$

Examination of each groups pain profiles provides evidence that specific points when palpated reproduced the pain and symptoms reported in CUP. These points provide evidence of the myofascial origin of pain, in contrast to the erroneous assumptions made in the past that pain was of diseased organ origin.

Assessment of points listed in Map A, identifies the vestibule and urethral meatus as two of the most sensitive structures. However, of the 27 points examined, only nine points (all relating to the vestibule and urethra), were reported as painful. Surprisingly, the Q-tip test of the vestibule showed that in asymptomatic women the genital area can also be sensitive and painful. The superficial fascia of Coles makes up the vestibular tissue, and as such causes localised discomfort but no referred pain. Only the richly innervated deep fascia refers pain to more distant areas. ${ }^{26}$ Women who typically report genital irritation triggered by wearing tight clothing, may 
be experiencing superficial fascial sensitivity. The Q-tip test which has been used for over 30 years, as a primary diagnostic tool with vulvodynia, only assess superficial sensitivity with insufficient attention being given to other potential sources of pain and symptoms that are typically associated with CPP and CUP. A Q-tip test provides limited information and may potentially lead to misleading conclusions.

Analysis of data from Map B shows that with the exception of the piriformis muscle, all points were rated as significantly painful. Each of these points differentiated between symptomatic and asymptomatic cases. In addition to pain at the point of palpation, these points referred pain to distant areas within and outside of the pelvis as shown in Figure 5B. Palpation of the deeper pelvic muscles - the iliococcygeus and puborectalis, referred pain to the anorectal area and reproduced a sense of bowel urge. The coccygeus and piriformis muscles referred pain to the lower back, buttocks and tailbone area, while the obturator internus muscle produced pain that radiated into the lower back, hip, abdominal quadrants and thighs. Given that these structures are associated with deep fascial tissue the referred pain patterns were much more extensive.

On Map C, the paraurethral points consistently provided the highest pain ratings of all the points mapped, making this the most painful anatomical region examined. Pain originating from the paraurethral area was often distressing and described as burning, sharp, stabbing and sometimes as itching. Mean pain scores in the paraurethral area increased from the distal to proximal section of the urethra, and pain scores on the left side of the urethra were higher than those on the right side.

The twelve points examined reproduced urethral and bladder pain, urge, clitoral pain, sensation of suprapubic pressure, referred pain to the umbilicus, pubic area, left and right inguinal quadrants, groin, gluteal area, lower lumbar region, and in some instances to the soles of the feet, as shown in Figure 5C. Examination of the paraurethral points reproduced many of the symptoms commonly reported by CUP cases. Yet, none of the symptomatic participants reported paraurethral pain prior to examination. The pain appears to be imperceptible, and a form of latent, passive pain, that is only reproduced by physical palpation of the area. This finding highlights the need for physical examination to follow a given protocol irrespective of the pain areas reported by a patient. This study provides direction and is key to the most relevant points for assessment.

The highest pain scores were noted on Map C and require some potential explanation. There are several reasons why this may be the case. The unusually high pain scores may be related to the density of innervation and vascularization of the anterior vaginal wall and urethral lumen. ${ }^{27}$ The upper two thirds of the urethra and anterior vagina are fused into a single structure. This structure is enveloped by the bulbo-clitoral organ and forms part of the highly sensitive female sexual complex. As such, it is an area that may be the primary source and cause of dyspareunia, which is poorly understood and unexplained in literature. Another reason for the high pain scores is that the posterior pubis is a significant point of tensional convergence for pelvic muscle and endopelvic fascia. As an anchoring point for the pubocervical fascia that spans the urogenital hiatus, and for the pelvic diaphragm and the ligaments that hold the urethra, bladder and vagina in place, it is prone to inflammation, fascial densification, increased sensitivity, pain and tensional dysregulation that impacts all organs in its proximity. Given that the muscles, ligaments and organs form one unified structure held together by the endopelvic fascia, this area may be highly reactive to any tensional changes.

In women who reported experiencing urinary urge, palpation of paraurethral points consistently reproduced the sensation of an irresistible desire to void. The sudden urge to void was often reported as causing more distress than the sharp, burning pain associated with palpation. Within the investing fascia are the extramural and intramural ganglia that regulate the micromotions and peristaltic movement of organs and glands. ${ }^{28}$ Since these ganglia are highly reactive to alterations in fascial tension, whether due to non-relaxing pelvic muscles, fascial restriction or palpation pressure, the sense of urge is consistently reproduced during physical examination. Dysregulation of bladder pacemaker action is a sign noted in $70 \%$ of BPS patients. ${ }^{16}$ Reproduction of these exaggerated sensations in the bladder, in the absence of any changes in intravesicular pressure, points to local mechanisms within investing fascia mediating bladder excitability.

A subgroup of CUP women reported experiencing clitoral pain, known as clitorodynia, a localised form of vulvodynia. Others reported symptoms of persistent genital arousal, a form of unwanted sexual arousal. ${ }^{29,30}$ Both of these symptoms were reproduced by palpation of the paraurethral points. Even among symptomatic women who did not specifically report clitoral pain, palpation of the area produced pain radiating into the clitoral glans. Given that the sensitive bulbo-clitoral organ surrounds the urethra and vagina the mechanisms of pain can be better understood. The fact that clitoral pain is only reproduced by paraurethral examination and not by a Q-tip test of the clitoral glans, illustrates that an organ can be an innocent bystander affected by referred pain but 
not be the source of pain. On the basis of pain mapping the mechanisms leading to clitoral pain and persistent genital arousal may be related to and arise from changes in soft tissue in the anatomical region but unrelated to any suspected disease process. Furthermore, clitoral pain and persistent genital arousal appear to be a part of the CUP continuum, originating from the paraurethral area, and do not constitute separate disorders.

\section{CONCLUSIONS}

Several important findings arise from this study with significant implications for the management of CPP and CUP. It is evident that the use of a consistent protocol for physical examination of pelvic structures and for the localisation of pain and symptoms is essential.

Using a validated pain mapping protocol facilitates consistent and accurate assessment. Pain mapping can be used in various formats. Logistic regression analysis showed that pain mapping can be used as a reliable diagnostic guide. Selecting just two points from each map can provide $94 \%$ accuracy of diagnosis. It can also be used in an abbreviated form for reliable assessment and establishing individual pain profiles. Otherwise, the extended form can be used for research purposes as was the case in this study.

The traditional Q-tip test provides limited information and should not be relied upon for diagnostic purposes. Limiting assessment to the superficial fascia of the vestibule does not explain the wide range of symptoms experienced by women with CUP. The deep fascia of the pelvic muscles and the paraurethral area appears to be the more critical mechanism by which referred pain is communicated throughout the body. Understanding the continuity of fascia which links the abdominal, pelvic and lumbar regions explains the mechanism by which abdominal, groin and lower back pain interlink with pelvic pain and organ dysfunction.

Another insight from this study is that pressure application to deeper tissue impacts on organ function. The sensation of bowel and bladder urge is potentially mediated by tensional changes that activate local ganglia involved in regulating peristalsis of the bowel and micromotions of the bladder. ${ }^{28}$ Tensional variations in visceral fascia may also be the mechanism by which so many of the pelvic disorders are comorbid to each other.

Vulvodynia, BPS and irritable bowel syndrome are the most common co-morbidities noted.

The fact that the mean Oxford scale score for the CUP group was significantly lower than the control group $[2.6( \pm 1.9)$ vs
3.5 ( \pm 1.7$)$ respectively], is most likely an indication of fatigue due to non-relaxing muscles seen in chronic pain cases. Muscle fatigue should not be confused with muscle weakness.

To date the lack of objective protocols for the assessment of CUP results in costly and invasive tests in search of non-existent pathology. Women often reported undergoing multiple laparoscopies and ultrasounds with negative findings. Clearly the focus was directed to the wrong causes and mechanism of pain. Likewise, cystectomies, cliterodectomies, vestibulectomies and the total removal of reproductive organs in young women, did not eliminate or reduce the severity of urogenital pain, again highlighting the fact that organs are not the source of pain. ${ }^{31,32} \mathrm{~A}$ number of recent studies examining the relationship between pain and endometriosis found no correlation between severity of pain and level of disease. Each of the studies recommended that pain of myofascial origin should be explored and addressed in therapy. $8,17,18$

The results of this pain mapping study provide essential information on the profiles of CUP syndromes, the mechanisms of pain, and pave the way for innovative interventions and therapies. Therapies guided by pain mapping assessments need to focus on fascial restrictions and densification of fascia which can occur in response to tissue trauma, scars, inflammation or emotional tension. Whatever the trigger may be, normalising muscles and mobilising facial tissue need to be the primary focus of therapy. Further research on pain mapping and profiling of specific disorders will provide knowledge and insights. One of the weaknesses of this study is that it compared only two CUP disorders and the numbers in the BPS group were relatively small. Larger samples should be used in the future. The pain profiles of various diagnostic groups need to be compared, including those of women who present with endometriosis. Many of these disorders need to be better understood from further mechanismms-oriented research.

\section{DISCLOSURES}

The author declares no conflict of interest, and no financial support by any grant or research sponsor.

Peer-review: Externally peer-reviewed.

\section{REFERENCES}

1. Howard FM. Chronic pelvic pain. Obstet Gynecol. 2003; 101: 594611.

2. Stein SL. Chronic pelvic pain. Gastroenterol Clin North Am 2013; 42 : 785-800.

3. Sedighimehr N, Manshadi FD, Shokoohi N, Baghban AA. Pelvic musculoskeletal dysfunction in women with and without pelvic pain. J Bodyw Mov Ther. 2018; 22: 92-6. 
4. Tu FF, As-Sanie S, Steege JF. Prevalence of pelvic musculoskeletal disorders in a female chronic pelvic pain clinic. J Reprod Med 2006; 5: 185-9.

5. Butrick CW. Pelvic hypertonic disorders: Identification and management. Obstet Gynecol Clin North Am 2009; 36: 707-22.

6. Predengarst SA, Rummer EH. Interdisciplinary management of chronic pelvic pain: A US physical medicine perspective. In: Chaitow L, Lovegrove Jones R, eds. Chronic pelvic pain and dysfunction: Practical physical medicine. London: Churchill Livingstone Elsevier; 2013: 171-84.

7. Meister MR, Shivakumar N, Sutcliffe S, Spitznagle T, Lowder JL. Physical examination techniques for the assessment of pelvic floor myofascial pain: a systematic review. Am J Obstet Gynecol 2018; 219: 497.

8. Aredo JV, Heyrana KJ, Karp BI, Shah JP, Stratton P. Relating chronic pelvic pain and endometriosis to signs of sensitization and myofascial pain and dysfunction. Sem Reprod Med 2017; 35: 88-97.

9. Fall M. Foreword. In: Chaitow L, Lovegrove Jones R, eds. Chronic pelvic pain and dysfunction: practical physical medicine. London: Churchill Livingstone Elsevier, 2013.

10. Kaufman R, Friedrich E, Gardner H. Non-neoplastic epithelial disorders of the vulvar skin and mucosa; miscellaneous vulvar disorders, benign diseases of the vulva and vagina. Chicago IL: Chicago Yearbook, 1989: 299-360.

11. Friedrich EG Jr. Therapeutic studies on vulvar vestibulitis. J Reprod Med 1988; 33: 514-8.

12. Bachmann GA, Rosen R, Pinn VW, et al. Vulvodynia: a state of the art consensus on definitions and management. J Reprod Med 2006; 51: 447-56.

13. Jantos M. Surface electromyography and myofascial therapy in the management of pelvic pain disorders. In: Santoro GA, Wieczorek P, Bartram C, eds. Pelvic floor disorders: imaging and a multidisciplinary approach to management Milan: Springer Verlag Italia, 2010: 593-607.

14. Jantos M. Electromyographic assessment of female pelvic floor disorders. In: Criswell E, ed. Cram's introduction to surface electromyography. 2nd ed. Sudbury: Jones \& Bartlett Publishers, 2010: 203-30.

15. Jantos M. Vulvodynia: a psychophysiological profile based on electromyographic assessment. Appl Psychophysiol Biofeedback 2008; 33: 29-38.

16. Peters KM, Carrico DJ, Diokno AC. Characterization of a clinical cohort of 87 women with interstitial cystitis/painful bladder syndrome. Urology. 2008; 71: 634-40.
17. Stratton P, Khachikyan I, Sinaii N, Ortiz R, Shah J. Association of chronic pelvic pain and endometriosis with signs of sensitization and myofascial pain. Obstet Gynecol. 2015; 125: 719-28.

18. Orr NL, Noga H, Williams C, et al. Deep dyspareunia in endometriosis: role of the bladder and pelvic floor. J Sex Med 2018; 15: 1158-66.

19. Reissing E, Brown C, Lord M, Binik YM, Khalife S. Pelvic floor muscle functioning in women with vulvar vestibulitis syndrome. J Psychosom Obstet Gynecol. 2005; 26: 107-13.

20. Kavvadias T, Pelikan S, Roth P, Baessler K, Schuessler B. Pelvic floor muscle tenderness in asymptomatic, nulliparous women: topographical distribution and reliability of a visual analogue scale. Int Urogynecol J. 2013;24;281-6.

21. Jantos M, Johns S, Torres A, et al. Mapping chronic urogenital pain in women: rationale for a muscle assessment protocol - the IMAP, Part 1. Pelviperineology. 2015; 34: 21-7.

22. Jantos M, Johns S, Torres A, Radomanska EB. Mapping chronic urogenital pain in women: insights into mechanisms and management of pain based on the IMAP, Part 2. Pelviperineology. 2015; 34: 28-36.

23. Hjermstad MJ, Fayers PM, Haugen DF, et al. Studies comparing numerical rating scales, verbal rating scales, and visual analogue scales for assessment of pain intensity in adults: a systematic literature review. J Pain Symptom Manage. 2011; 41: 1073-93.

24. Melzack R. The McGill pain questionnaire: from description to measurement. Anesthesiology. 2005; 103: 199-202.

25. Johns S. Development and validation of a chronic urogenital pain mapping tool for women: insights derived for understanding mechanisms of pain and implications for management. PhD thesis. School of Nursing and Midwifery, University of South Australia, 2017.

26. Jantos M, Stecco C. Fascia of the Pelvic Floor. In: Schleip R, Driscoll $M$, Stecco C, Huijing P, eds. Fascia: the tensional network of the human body. 2nd ed. Churchill: Livingstone Elsevier, 2020.

27. Di Marino V, Hubert L. Anatomical study of the clitoris and the bulbo-clitoral organ. Springer, 2014.

28. Stecco L, Stecco C. Facial manipulation for internal dysfunctions. Piccin, Italy: Padova, 2014.

29. Waldinger MD, Schweitzer DH. Persistent genital arousal disorder in 18 Dutch women: Part II. A syndrome clustered with restless legs and overactive bladder. J Sex Med 2009; 6: 482-97.

30. Waldinger MD, Venema PL, Van Gils AP, Schutter EM, Schweitzer $\mathrm{DH}$. Restless genital syndrome before and after clitoridectomy for spontaneous orgasms: A case report. J Sex Med 2010; 7: 1029-34.

31. Baskin LS, Tanagho EA. Pelvic pain without pelvic organs. J Urol 1992;147:683-686.

32. Brookoff D. Genitourinary pain syndromes: Interstitial cystitis, chronic prostatitis, pelvic floor dysfunction, and related disorders. In: Smith H, (ed). Current Therapy in Pain. Saunders Elsevier, Philadelphia, 2009. p. 205-215. 